\title{
Transverse polarization light scattering in tubular semiconductor nanowires
}

\author{
Miguel Urbaneja Torres*, Anna Sitek ${ }^{*, \ddagger}$, and Andrei Manolescu* \\ ${ }^{*}$ School of Science and Engineering, Reykjavik University, Menntavegur 1, IS-101 Reykjavik, Iceland \\ ${ }^{\ddagger}$ Department of Theoretical Physics, Faculty of Fundamental Problems of Technology, Wroclaw University of \\ Science and Technology, 50-370 Wroclaw, Poland \\ e-mail:miguelt16@ru.is
}

\begin{abstract}
We carry out numerical calculations of the scattering cross section of tubular semiconductor nanocylinders in the optical range. The scattering is investigated for the transversal incidence of light, i.e., along the diameter of the cylinder, with both transverse electric and transverse magnetic polarization. These subwavelength nanostructures support Mie resonances and, when the length of the cylinder is comparable to the wavelength, guided modes that can overlap with the Mie modes giving rise to sharp Fano resonances. We show that a varying internal radius affects each mode differently, allowing for an extra degree of freedom for tuning the spectral position of the resonant peaks.
\end{abstract}

Keywords: semiconductor nanowires, nanotubes, light scattering, Mie modes.

\section{INTRODUCTION}

The study of light-matter interaction in dielectric nanostructures with high refractive index has recently become one of the most attracting research topics in photonics [1]. The interest in these structures stems from their ability to scatter light in the optical range with, unlike their plasmonic metallic counterparts, almost negligible losses [2] while being able to support multiple resonant modes. This makes them great candidates for building blocks of optoelectronic devices capable of manipulating visible light at the nanoscale [3]. The magnetic and electric resonances coexist within the same spectral domain and are highly tunable through the material and the structure geometry [4]. A wide range of structures, ranging from simple spherical particles [5], and cylindrical nanowires [6, 7], to other shapes including blocks or pyramids [8] and metasurfaces [9], have been studied and showed interesting phenomenology with original applications. These include directional scattering through the interference of different resonant modes [10], light propagation via near-field coupling between mesoscopic scatterers [11], second and third-harmonic generation [12], or Raman scattering [13].

Additionally, Fano resonances, which are asymmetric line-shape scattering resonances, have also attracted considerable attention in the last years [14], due to their promising applicability in optical switching and sensing. In the case of dielectric materials Fano resonances are typically obtained in dimers and oligomers. However, it has recently been shown that strong Fano resonances can also occur in simple-shaped single dielectric nanowires [15] where they are the result of the interference between a sharp guided mode and either a broad leaky mode or a Mie background.

Analytical calculations of the absorption and the scattering of light due to nanostructures are possible only for spherical particles (the so called Mie problem) and for infinite cylinders [16]. In our present work, we consider hollow semiconductor nanowires, i.e., nanocylinders or nanotubes, and evaluate the scattering cross section under transversal electric or magnetic (TE or TM) plane waves illumination using numerical methods. We first carry out calculations for short nanowires and show how the tubular structure affects the resonant Mie modes, in particular we study their dependence on the internal radius. Next we repeat the simulations for longer nanowires in order to study the effects on the Fabry-Pérot guided modes and on the Fano resonances. We show that, for TE polarization, a varying internal radius strongly affects the Mie modes whereas the Fabry-Pérot guided modes are almost unaffected. These properties allow to obtain different overlapping, and thus enable the control of the Fano resonances.

\section{METHODS}

We carry out numerical calculations of the scattering cross section using finite element method (FEM) and perform the simulations using Comsol Multiphysics 5.4 (wave optics module) [17]. In order to optimize the calculation time we take the advantage of the radial symmetry. The simulation consists of a quadrant of a sphere with $1200 \mathrm{~nm}$ radius in which a quarter of the nanowire, cut by two symmetry axes, is embedded in the center. The sphere consists of an outer perfectly-matched layer (PML) of $400 \mathrm{~nm}$ thickness and the inner space where the nanowire is located. We impose boundary conditions (perfect electric conductor [PEL] and perfect magnetic conductor $[\mathrm{PMC}]$ ) on the facets of the quadrant for the background electromagnetic field according to the chosen 

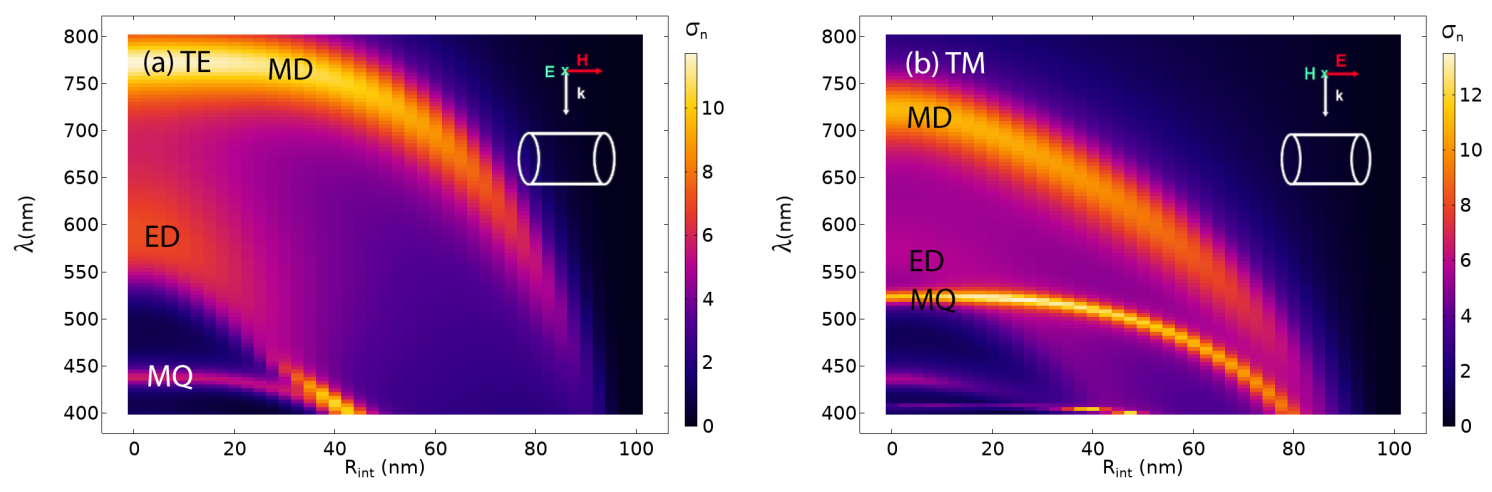

Figure 1: Normalized scattering cross section spectral dependence on internal radius $R_{\text {int }}$ for a short nanowire under TE polarization (a) and TM polarization (b). In both cases the nanowire length is $L=150 \mathrm{~nm}$.

polarization of the incident light. We first consider short cylinders, with the length of $L=150 \mathrm{~nm}$, and the external radius of $R_{\text {ext }}=100 \mathrm{~nm}$. Our cylinders have a tubular shape, with a constant external radius $\left(R_{\text {ext }}\right)$ and the internal one $\left(R_{\text {int }}\right)$ varying between zero and $R_{\text {ext }}$. We study the spectral dependence of the normalized scattering cross section of light in the range of $\lambda=400-800 \mathrm{~nm}$. Further, we consider longer nanowires, i.e., of length $L=600 \mathrm{~nm}$, and repeat the calculations for the scattering cross section for the incident light wavelengths up to $\lambda=1000 \mathrm{~nm}$. Finally, we obtain the spectra for the constant value of the aspect ratio $R_{\text {int }} / R_{\text {ext }}=1 / 2$, and variable external diameter $\left(2 R_{\text {ext }}\right)$. We compare the results with the spectra obtained for the bulk nanowire. In all cases the refractive index is fixed to $n=3.5$, which corresponds to $\mathrm{GaP}$ at $\lambda=530 \mathrm{~nm}$ [18], and we assume that the nanowires are surrounded by air $(n=1)$. Since we are mostly interested in the effects of the geometry, the absorption is neglected in all the cases. The results are still valid and scalable for materials with negligible dispersion in the refractive index, such as $\mathrm{GaP}$ in the optical range or $\mathrm{Si}$ in the infrared domain.

\section{RESULTS}

\subsection{Short nanowires}

First we study the scattering cross section of short cylindrical particles in which a longitudinal hole of increasing radius is created. Such hollow dielectric particles, whose dimensions are smaller than the light wavelength, are known to support strong magnetic or electric Mie resonances. A normally incident plane wave may excite either vertical TE or TM modes [7].

As expected, with increasing the internal radius of the tubular structure, the resonant scattering modes are blueshifted, because the thickness of the cylindrical shell is reduced, and thus the effective scattering volume shrinks. However, not all the modes decay with the same rate. As shown in Fig. 1 under TE polarization, the electric dipole (ED) shifts remarkably faster than the magnetic dipole (MD) and the magnetic quadrupole (MQ). The MD, oriented along the axis of the cylinder, is almost unaffected for $R_{\mathrm{int}}<40 \mathrm{~nm}$, since the displacement currents that create it are stronger on the outer part of the nanowire. Under TM polarization both the ED and the MD are shifted in the same way. We also observe that the MD mode predominates when the impinging light is TE polarized whereas under TM polarized light the MQ becomes the dominant mode. A detailed explanation of the behavior of these modes can be found in [7].

\subsection{Long nanowires}

As the length of the nanowires increases, the resonant Mie modes evolve until they converge to those of an infinite cylinder. These transverse resonances may coexist with such longitudinal guided modes as Fabry-Pérot resonances start to develop as a function of the length. This is the result of the transverse modes bouncing back and forth along the length of the nanowire. These overlapping of transverse and longitudinal modes leads to sharp Fano resonances [15].

Here we explore how to tailor the Fano resonances by changing the geometry of the nanowires. The presence of the longitudinal hole distorts the Mie transverse modes in the same way as for the short nanowires studied in the previous section. However, the guided modes are affected in a different way. As shown in Fig. 2 under TE incidence, the guided modes $\left(\mathrm{TE}_{01}\right)$ are almost unaffected by the increasing internal radius since they are associated to the MD. This situation allows for the inversion of the symmetry of the Fano resonances because the blue-shift of the broad ED mode is much faster. On the other hand, under TM polarization both the Mie background and the 
hybrid guided modes $\left(\mathrm{HE}_{11}\right)$ are blue-shifted at equivalent rates, and thus the crossing of the main resonant peaks is avoided.
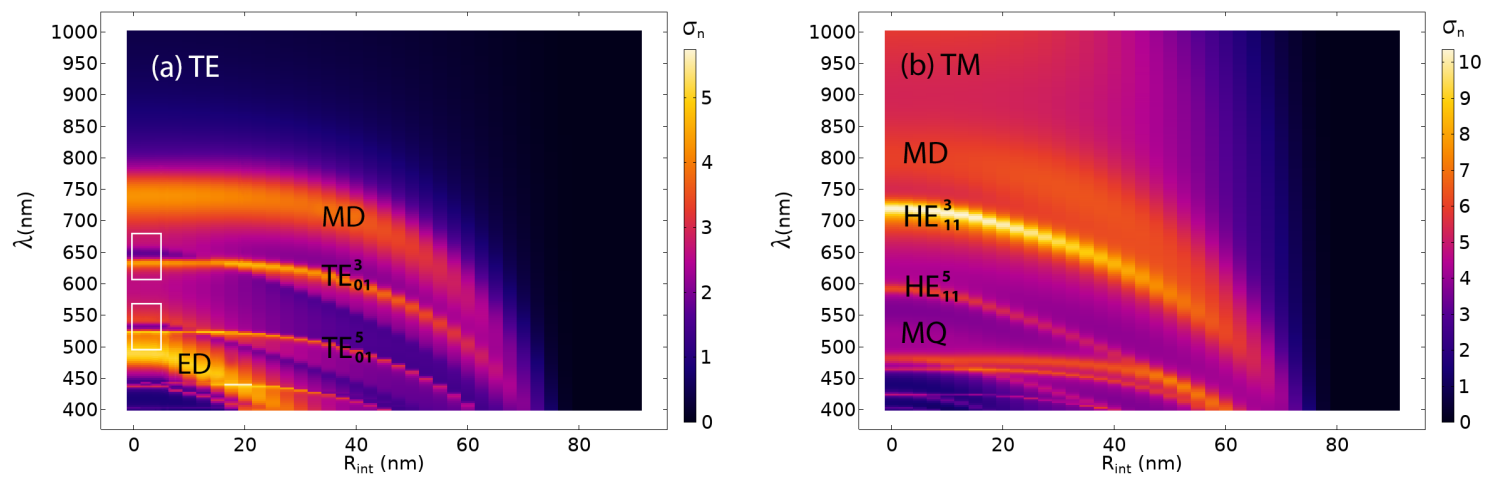

Figure 2: As in Fig. 1 but for $L=600 \mathrm{~nm}$. The rectangles indicate the ranges where the main Fano resonances occur.
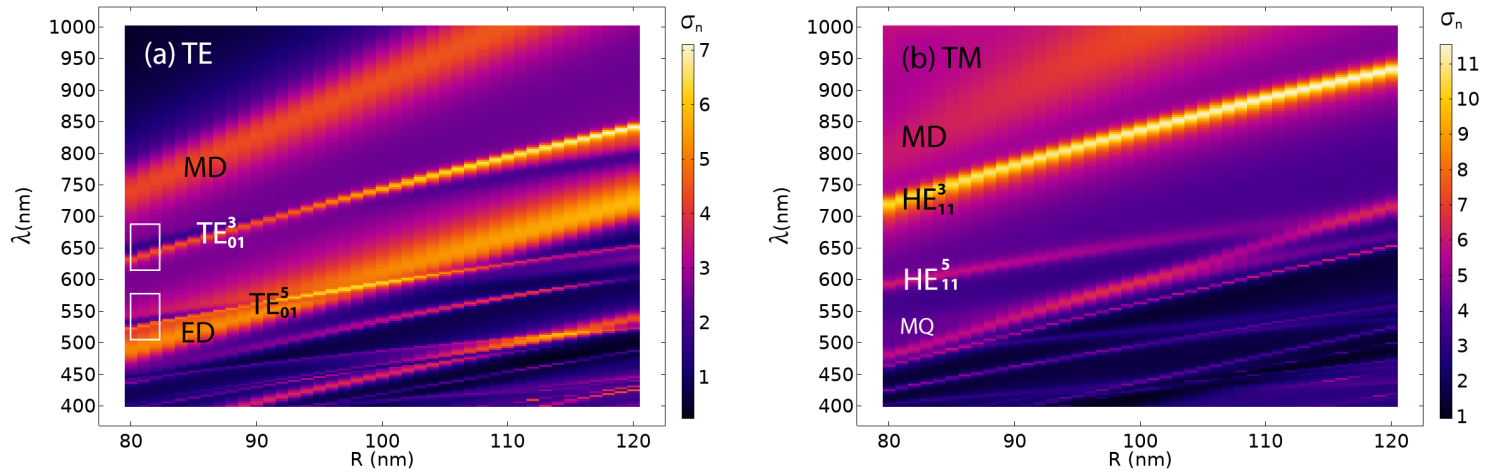

Figure 3: Normalized scattering cross section spectral dependence on external radius $R_{\text {ext }}$ for a (full) cylindrical nanowire under TE polarization (a) and TM polarization (b). In both cases the length is $L=600 \mathrm{~nm}$.
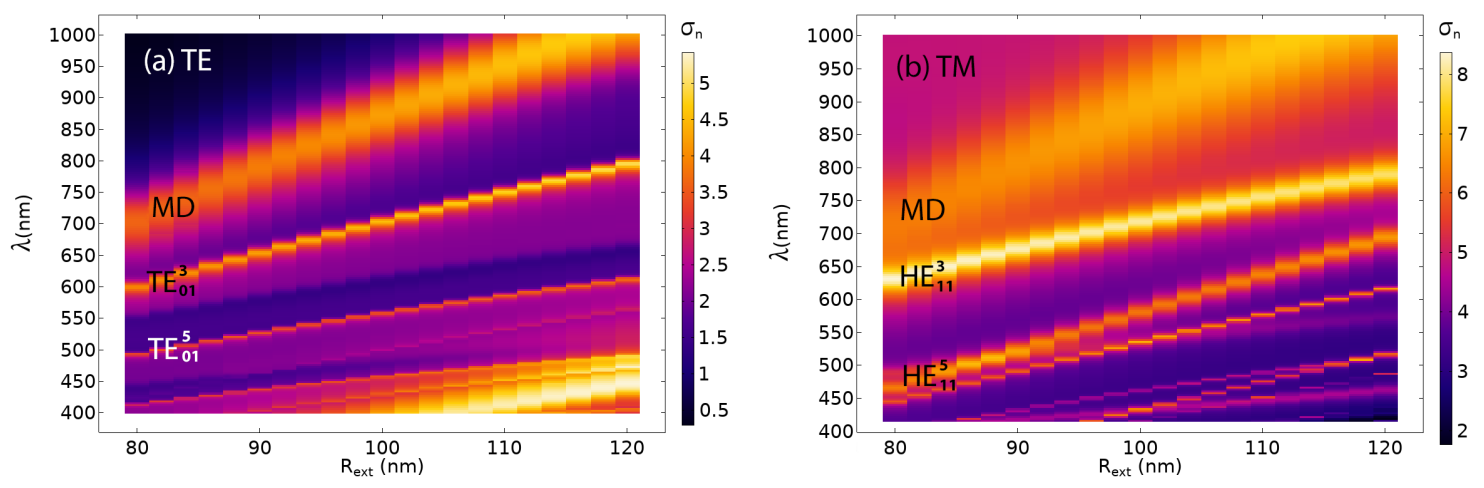

Figure 4: Normalized scattering cross section spectral dependence on the external radius $R_{\text {ext }}$ for a tubular nanowire with a fixed ratio $R_{\text {int }}=R_{\text {ext }} / 2$. In both cases the length $L=600 \mathrm{~nm}$.

A detailed description of these modes for cylindrical nanowires, i.e., for which $R_{\text {int }}=0$, can be found in [15]. Here we also compare the dependence of the scattering spectra on the external radius for cylindrical (Fig. 3) and tubular nanowires (Fig. 4 with fixed aspect ratio $\left(R_{\text {int }}=R_{\text {ext }} / 2\right)$. Now the scattering cross section increases with the radius, and thus all the modes are red-shifted and their spectral position depends on the size parameter $x=\pi R_{\text {ext }}^{2} / \lambda$. Within the studied range of external radii $\left(R_{\text {ext }}=80-120 \mathrm{~nm}\right)$ the modes are linearly red shifted with different rates. In the case of a full nanowire exposed to TE polarized light, the increasing radius creates a crossing of modes, which again allows for an inversion of the Fano resonance. However, for the tubular nanowire, the red-shifting rate of the resonant modes does not differ much between the Mie background and the guided 
modes, again avoiding any crossing in the studied range.

\section{CONCLUSIONS}

We have studied the geometrical optical resonances and calculated the scattering cross section of semiconductor cylindrical nanowires and the transition from bulk to tubular cylinders. We have shown that the tubular structure offers an additional degree of freedom for tuning the spectral position of the resonant modes. Since various modes are affected in different ways by the geometrical parameters it is possible to invert Fano resonances and change the spectral spacing between modes. This possibility is explored both in full nanowires by changing the external radius and in tubular structures by changing the internal radius.

\section{ACKNOWLEDGEMENTS}

This work was financed by the Icelandic Research Fund, project 163438-051.

\section{REFERENCES}

[1] Arseniy I. Kuznetsov, Andrey E. Miroshnichenko, Mark L. Brongersma, Yuri S. Kivshar and Boris Luk'yanchuk, Optically resonant dielectric nanostructures, Science 354, 6314 (2016).

[2] P. Albella, R. Alcaraz de la Osa, F. Moreno and S. A. Maier, Electric and Magnetic Field Enhancement with Ultralow Heat Radiation Dielectric Nanoantennas: Considerations for Surface-Enhanced Spectroscopies, ACS Photonics 1, 524 (2014).

[3] Arseniy I. Kuznetsov, Andrey E. Miroshnichenko, Yuan Hsing Zhang Fu, Bo Jing and Boris Luk'yanchuk, Magnetic light, Scientific Reports 2, 492 (2012).

[4] J. van de Groep and A. Polman, Designing dielectric resonators on substrates: Combining magnetic and electric resonances, Opt. Express 21, 26285 (2013).

[5] A. Garcia-Etxarri, R. Gomez-Medina, L. S. Froufe- Pérez, C. López, L. Chantada, F. Scheffold, J. Aizpurua, M. Nieto-Vesperinas, and J. J. Sáenz, Strong magnetic response of submicron Silicon particles in the infrared, Opt. Express 19, 4815 (2011).

[6] L. Cao, P. Fan, E. S. Barnard, A. M. Brown, and M. L. Brongersma, Tuning the Color of Silicon Nanostructures, Nano Letters 10, 2649 (2010).

[7] M. A. van de Haar, J. van de Groep, B. J. Brenny, and A. Polman, Controlling magnetic and electric dipole modes in hollow silicon nanocylinders, Opt. Express 24, 2047 (2016).

[8] A. B. Evlyukhin, C. Reinhardt, and B. N. Chichkov, Multipole light scattering by nonspherical nanoparticles in the discrete dipole approximation, Phys. Rev. B 84, 235429 (2011).

[9] Y. Yang, W. Wang, P. Moitra, I. I. Kravchenko, D. P. Briggs, and J. Valentine, Dielectric Meta-Reflectarray for Broadband Linear Polarization Conversion and Optical Vortex Generation, Nano Letters 14, 1394 (2014).

[10] S. Person, M. Jain, Z. Lapin, J. J. Sáenz, G. Wicks, and L. Novotny, Demonstration of Zero Optical Backscattering from Single Nanoparticles, Nano Letters 13, 1806 (2013).

[11] R. R. Naraghi, S. Sukhov, J. J. Sáenz, and A. Dogariu, Near-Field Effects in Mesoscopic Light Transport, Phys. Rev. Lett. 115, 203903 (2015).

[12] Z.-J. Yang, Fano Interference of Electromagnetic Modes in Subwavelength Dielectric Nanocrosses, The Journal of Physical Chemistry C 12021843 (2016).

[13] M. Caldarola, P. Albella, E. Cortés, M. Rahmani, T. Roschuk, G. Grinblat, R. F. Oulton, A. V. Bragas, and S. A. Maier, Non-plasmonic nanoantennas for surface enhanced spectroscopies with ultra-low heat conversion, Nature Communication 6, 7915 (2015).

[14] F. M. Limonov, V. M. Rybin, N. A. Poddubny, and S. Y. Kivshar, Fano resonances in photonics, Nature Photonics 11, 543 (2017).

[15] D. R. Abujetas, M. A. G. Mandujano, E. R. Méndez, and J. A. Sánchez-Gil, High-Contrast Fano Resonances in Single Semiconductor Nanorods, ACS Photonics 4, 1814 (2017). 
[16] C. F. Bohren and B. R. Huffman, Absorption and Scattering of Light by Small Particles, Wiley-VCH (2004).

[17] COMSOL Multiphysics @ v. 5.4. www.comsol.com. COMSOL AB, Stockholm, Sweden.

[18] D. E. Aspnes and A. A. Studna, Dielectric functions and optical parameters of Si, Ge, GaP, GaAs, GaSb, InP, InAs, and InSb from 1.5 to $6.0 \mathrm{eV}$, Phys. Rev. B 27, 985 (1983). 